\title{
Application of genotyping by sequencing technology to a variety of crop breeding programs
}

Changsoo Kim, Hui Guo, Wenqian Kong, Rahul Chandnani, Lan-Shuan Shuang, Andrew H Paterson $^{1}$

Plant Genome Mapping Laboratory, University of Georgia, Athens, GA 30602, USA

${ }^{1}$ Corresponding author:

Andrew H. Paterson

Plant Genome Mapping Laboratory

University of Georgia

111 Riverbend Road, Rm 228,

Athens, GA 30602

USA

Phone 1-706-583-0162; Fax 1-706-583-0160; Email paterson@uga.edu

(C) 2015. This manuscript version is made available under the Elsevier user license http://www.elsevier.com/open-access/userlicense/1.0/ 


\begin{abstract}
Since the Arabidopsis genome was completed, draft sequences or pseudomolecules have been published for more than 100 plant genomes including green algae, in large part due to advances in sequencing technologies. Advanced DNA sequencing technologies have also conferred new opportunities for highthroughput low-cost crop genotyping, based on single-nucleotide polymorphisms (SNPs). However, a recurring complication in crop genotyping that differs from other taxa is a higher level of DNA sequence duplication, noting that all angiosperms are thought to have polyploidy in their evolutionary history. In the current article, we briefly review current genotyping methods using next-generation sequencing (NGS) technologies. We also explore case studies of genotyping-by-sequencing (GBS) applications to several crops differing in genome size, organization and breeding system (paleopolyploids, neo-allopolyploids, neo-autopolyploids). GBS typically shows good results when it is applied to an inbred diploid species with a well-established reference genome. However, we have also made some progress toward GBS of outcrossing species lacking reference genomes and of polyploid populations, which still need much improvement. Regardless of some limitations, low-cost and multiplexed genotyping offered by GBS will be beneficial to breed superior cultivars in many crop species.
\end{abstract}

Key words: genotype-by-sequencing, marker-assisted selection, genetic study, genomics

\title{
Contents
}

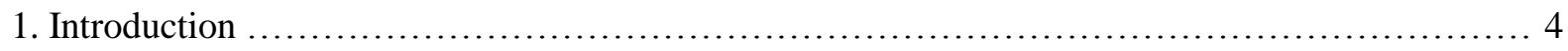

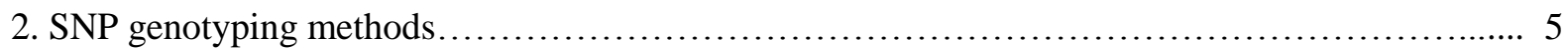

2.1. Array- or PCR-based SNP genotyping platforms............................... 5

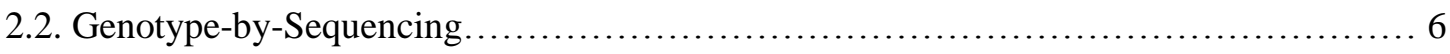




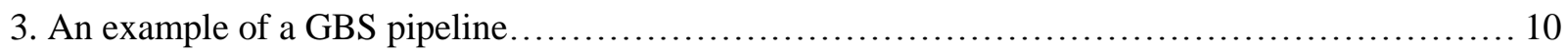

3.1. GBS protocol and data analysis........................................... 10

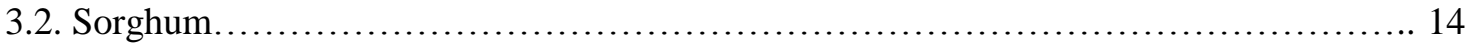

3.3. Brassica............................................................. 15

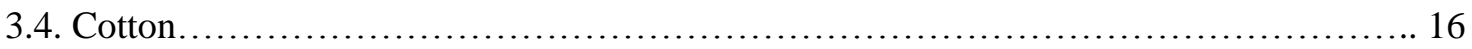

3.5. Miscanthus................................................................. 16

4. Future perspectives and concluding remarks......................................... 17

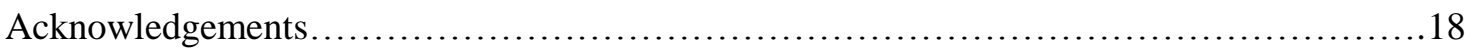

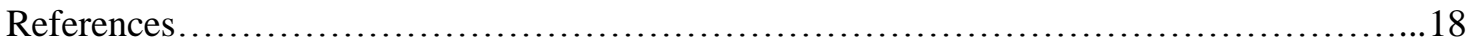




\section{Introduction}

One of the global key phrases in the $21^{\text {st }}$ century is feeding the world. However, foreseeable climate changes can dramatically reduce agricultural productivity, mainly due to abiotic and biotic stresses. According to a recent report, the world population will not be stabilized in this century and will grow to 12.3 billion in 2100 [1]. With limited agricultural land, innovative crop cultivation together with the breeding of improved cultivars may be required for resolving the largest part of food security issues.

Plant breeding generally requires the analysis of the genetic background of a target species. In conventional breeding, farmers or breeders utilized phenotypic markers for selecting desirable plants; however, the use of molecular markers has dramatically changed the landscape of plant breeding. Molecular markers have a number of advantages in plant science in that they are (1) not subject to environmental influence, (2) theoretically available in vast numbers, and (3) usually more objective than phenotypic markers. Consequently, molecular markers can be used for marker-assisted selection (MAS) to greatly increase the efficiency and precision of plant breeding compared to conventional methods.

The use of MAS in plant breeding programs has been accelerated since the advent of the genomics era. Since the complete genomes of Arabidopsis thaliana [2] and Oryza sativa [3] were released, approximately 90 angiosperm genomes have been completed (as of December 2014, CoGePedia, http://genomevolution.org). A plethora of genome data has facilitated the application of sequence-based markers such as simple sequence repeats (SSR) and single nucleotide polymorphisms (SNP). The frequent use of genomic resources for plant breeding created a new term, genomics-assisted breeding $(\mathrm{GAB})$, integrating all genomics tools and resources. SNPs are abundant in a plant genome but have previously been costly and time-consuming for application to plant breeding programs. Recently, the cost and time were dramatically reduced by a variety of high-throughput SNP genotyping platforms, which expanded options available to plant breeders for utilizing abundant SNP markers in their programs. 
Advances in next-generation sequencing (NGS) technologies have taken the implementation of SNPs for genetic analysis to a new level. Genotype-by-Sequencing (GBS) has provided new opportunities for breeders with cost-effective, genome-wide scanning, and multiplexed sequencing platforms. In principle, GBS can simultaneously perform SNP discovery and genotyping, which is particularly advantageous for understudied species that lack reference genome sequences. The term GBS was first introduced to plant science by Elshire et al [4] while the method had been already available since the earliest form of GBS methods such as RAD-seq (restriction-associated DNA sequencing) [5]. Together with RAD-seq, the latest customized sequence-based SNP genotyping platforms [4, 6-10] have been intensively used for genetic mapping and association studies combined with phenotype data, providing the basis of reliable marker-trait relationships.

Here, we present the application of GBS for breeding purposes, with some case studies. While GBS has a number of advantages compared to conventional SNP genotyping platforms, it is still challenging in specific plants such as polyploids and outcrossing species. Thus, we also discuss future perspectives regarding these challenges.

\section{SNP genotyping methods}

\subsection{Array- or PCR-based SNP genotyping platforms}

Before the establishment of NGS technologies, SNP discovery and genotyping with DNA sequencing was extremely expensive and complicated. However, SNPs have a number of advantages such as their flexibility, speed and cost-effectiveness, making SNPs the primary choice for many genetic studies. While simple sequence repeats (SSRs), widely used before the SNP era, are more informative in terms of more frequently having multiple alleles per locus, the high information content sometimes limits their applications for genetic studies. For example, SSR data from different platforms or populations can be difficult to integrate due to variable information contents according to data sets. Also, SSR motifs are finite in a genome and not evenly distributed; the saturation of specific genomic regions may not be 
feasible. Most importantly, gel-based genotyping of SSRs is very laborious and automated fragment analysis systems are generally low-throughput even if they provide a moderate level of multiplexing. On the other hand, SNP markers can be easily converted to universal genotype information from different sources, permitting more powerful analyses of integrated SNP sets in a particular species. Although there are also some limitations of SNPs (discussed later), various concepts were adopted and used for SNP genotyping from PCR-based to higher throughput array-based platforms. Table 1 summarizes some important commercial SNP-genotyping platforms and their properties. Non-NGS-based platforms are high-throughput thanks to the high level of multiplexing. By using these platforms, cost per data point is less than SSR-based genotyping, but the SNP information of subject organisms is a prerequisite for designing a genotyping project, resulting in increased cost and longer experimental time. Nonetheless, these platforms are still useful tools when combined with economical SNP discovery pipelines using NGS technologies.

\subsection{Genotype-by-Sequencing}

NGS technologies have been game changers for genomic and genetic studies owing to their reduced cost of nucleotide sequencing. Advanced sequencing technologies contributed to the completion of the whole genome sequences of about 90 angiosperms that are being actively used as references for further research. Whole genome sequence data can be an ideal tool to call SNP variants, and was used to genotype SNPs for crop species with a reference genome (rice for example) [11-13], but low coverage and high sequencing cost per useful data point have been the biggest obstacle for this approach. Because it is not necessary or practical to generate deep whole-genome sequence data solely for genotyping purposes, several approaches have been applied to decrease the cost of NGS to a level where it can be implemented for SNP genotyping. Recently, the reduced representation library (RRL) has been widely adopted as an efficient approach to genotyping using NGS technologies [14]. The core component of the RRL approach is cutting the entire genome with specific restriction enzyme(s) to reduce genome complexity for the 
organism of interest. Since RRL targets a subset of a genome, the resulting sequence dataset can provide higher read coverage per locus while allowing higher level of multiplexing with uniquely bar-coded adapters for different samples. However, important genomic regions may not be captured by GBS libraries when restriction sites are not available surrounding those regions. Multiple GBS libraries with different combinations of enzymes may be able to overcome this issue. Different methods of GBS are listed in Table 2 with their specific features for technical comparisons.

To date, a large volume of plant research performed by GBS is available in scientific journals (reviews available by Poland and Rife, Deschamps et al, and He et al. [15-17]). Most studies have been conducted for species with reference genomes because SNP genotyping becomes much easier when a reference genome is available. The common steps after generating NGS reads are de-multiplexing and aligning them to a reference genome using a variety of multiple alignment tools. Bowtie [18] and BurrowsWheeler-Aligner (BWA) [19] are frequently used tools for GBS reads, generating SAM or BAM output files. The alignment information will be processed by actual genotyping pipelines such as MSG [6], Stacks [20] or TASSEL-GBS [21]. In case of non-model plant species, UStacks [20] and UNEAK [22] embedded in the Stacks and TASSEL-GBS, respectively, can be used but they generally require higher read coverage and stringent filtering steps to remove false positive SNPs.

GBS does not require a priori genomic knowledge of the species of interest, as it simultaneously conducts SNP discovery and genotyping. Therefore, GBS overcomes the shortcomings of conventional PCR- or array-based SNP genotyping methods and requires much less cost and time to obtain a critical mass of data. However, GBS still has room for improvement to be a general tool for a variety of genetic and genomics studies. Basically, GBS utilizes very low coverage of NGS reads so that it can accommodate as many multiplexed samples as possible. Low coverage frequently results in a number of genotyping errors. Sometimes, sequencing errors also result in false genotypes. Fig. 1 illustrates two types of common mistake caused by sequencing error or low read coverage of GBS. In fact, genotyping mistakes caused by 
sequencing errors (Fig. 1A) are not a big problem because GBS generally uses sequences with fairly high quality. In case of TASSEL-GBS, tag formation and genotyping use the first 64 bases of reads which are considered almost error-free regions. However, genotyping errors by low coverage regions (Fig. 1B) could be quite an issue for identifying homozygotes from heterozygotes. This problem could be worse in the genotyping of outcrossing species using the two way pseudo-testcross model [23]. In the same context, recombinant inbred line populations (RILs) are by far the most efficiently processed by GBS because of the predominance of homozygosity in these populations. This issue can be resolved by increasing sequencing depth either by using rare cutters, reducing the number of multiplexed samples in a library, or sequencing a library with higher capacity. These kinds of problems can be even worse when the reference genome of a target plant is not available. Plants are usually paleopolyploids that may have paralogous segments in the genome. Without reference genomes, these paralogs may be recognized as the same reads when similarities are very high. As stated, network-based SNP discovery pipelines such as UNEAK and UStacks could be used for genotyping non-model species, yet they are not as efficient as reference-based SNP discovery pipelines.

Many agriculturally important crops such as wheat, cotton, potato, sugarcane, and coffee are polyploids. Despite their importance, genetic studies using molecular markers have been constrained by their complex inheritance. While SNPs are not straightforward for genotyping polyploids due to their biallelic nature, plentiful genome-wide SNPs may be able to give new insight into genetic studies of polyploids. Traditionally, the study of polyploids has used simplex markers segregating like in diploid species. SNPs discovered by GBS can be used as simplex markers but much improvement is needed to resolve true alleles from paralogous reads. Like diploids, the accuracy of GBS in polyploids is relatively high with reference genomes. But, when reference genomes are not available problems can be even worse in GBS application to polyploids than diploids. Fig. 2 shows the complication of GBS data in tetraploids. GBS in autotetraploids may be a bit easier than allotetraploids because 'alloalleles' (at different loci that do not normally pair and recombine) need not be distinguished from multiple alleles that show polysomic 
inheritance. In autotetraploids, allelic dosage of each locus cannot be evaluated with low coverage GBS data but all the mixed-allele loci are genotyped as heterozygous (Fig. 1A). For accurate genotyping, those heterozygous loci in autotetraploids must be removed and fairly high coverage of GBS data is needed; however, the choice of homozygous loci may result in wasting a number of NGS reads. On the other hand, allotetraploids could be more complicated because disomic inheritance is expected in each subgenome. If a reference genome is available and the respective subgenomes are somewhat differentiated in DNA sequence, genotyping and mapping can be separated according to subgenomes. In contrast, two subgenomes with high similarity may not be readily distinguished from one another (Fig. 2B), and their genotyping may not be accurate even if heterozygous loci are removed. Thus, allotetraploid genotyping without reference genomes requires that sufficient variation exists to readily differentiate homeologous loci in different subgenomes.

Despite challenges to GBS of polyploids, genetic mapping with GBS has been carried out in allopolyploid grasses without reference genomes, to varying degrees. In switchgrass, linkage groups were defined with MMC clustering method [24], but ordering markers was not possible de novo because its outcrossing nature further complicated genotyping [22], instead relying on the genome of foxtail millet [25]. In the case of oat, a RIL population was used for GBS mapping [26]. Although the Hiseq 2500 technology (the highest sequencing capacity at the time) with 95 genotype multiplexing was used, only about 1000 SNPs were scattered to 35 linkage groups (versus the expected 21 linkage groups). A genetic map of a doubled haploid wheat population was fairly well constructed with a bin-mapping procedure with homozygous genotypes only [7]. These results suggest that GBS can be a useful tool to map polyploids to conduct marker-trait analysis for crops without reference genomes.

In partial summary, GBS is a useful application of NGS technologies which can be expanded to direct application in breeding programs. In particular, GBS is promising for orphan crops for which little genetic or genomic information is available, because discovering and genotyping SNPs can be performed 
simultaneously. The economy of GBS is dependent upon sequencing and bioinformatics cost. Although the cost of bioinformatics has not matched the dramatic decline in sequencing costs the last few years, advanced sequencing technologies have made it cost-effective and many methods have been reduced to routine practice. Plant breeders generally need rapid, cost-effective, and widely applicable diagnostic tools for genotyping a number of breeding populations. High throughput GBS data can deal with large scale breeding programs at the whole genome level. Genomics-assisted breeding (GAB) is a breeding concept which uses integrated genomics tools, using GBS to scan whole genomes with plenty of SNPs as well as focus on densely mapping specific genomic regions, facilitating the discovery of candidate genes. Despite some needs for improvement discussed in this section, it is envisioned that GBS will serve as a central element in future breeding programs.

\section{An example of a GBS pipeline}

\subsection{GBS protocol and data analysis}

Implementing a GBS protocol has allowed our group to expand and accelerate our crop genetics and genomics research. Our GBS is a slightly modified version of MSG [6] combined with the TASSEL-GBS [21] analysis pipeline. The basic sequencing platform is the Illumina MiSeq (equipped in-house) that generates up to 25 million reads of 150 base pairs (bps) fragments per run with single-end sequencing. The total number of reads per run may be insufficient to map high-density SNP sets compared to previous studies using HiSeq platforms, but with reduced genome complexity it is generally sufficient for genome scans of segregating populations in strong linkage disequilibrium such as F2, backcross or RIL populations requiring less than 1,000 SNPs for linkage-based QTL mapping. As needed, multiple sequencing reactions could capture additional read depth for genotyping.

The data processing pipeline consists of three major steps: 1) preprocessing of Illumina raw reads, 2) producing raw hapmap data using the TASSEL-GBS pipeline and 3) post processing of hapmap data according to mapping populations. 


\subsubsection{Preprocessing of Illumina raw reads}

For bi-parental populations, it is critical to minimize the number of missing values in parental lines to genotype mapping individuals. If parental lines are not already sequenced, then in order to increase the number of reads, parental genotypes are usually tagged with multiple barcodes in a multiplexed library. The NGS reads from each parental line with multiple barcodes are combined in silico. Since we currently have a 96-barcoding system, multiple libraries are constructed and sequenced for populations with larger than 96 individuals. The barcodes are located in the first 6 bases of raw reads. Therefore, reads that start with ' $\mathrm{N}$ ' and with no barcode in the first six bases are removed from further process. The processed reads are used as the input of TASSEL-GBS pipeline.

\subsubsection{TASSEL-GBS pipeline}

The TASSEL-GBS pipeline [21] includes both reference based and non-reference based genotype callers. If available, reference genome sequence can be used to guide the stacking of short reads that are from the same genomic location. TASSEL-GBS pipeline uses the first $64 \mathrm{bps}$ of the reads to minimize the effect of sequencing errors. The unique 64 bps reads (tags) are aligned to the reference genome using BWA [19]. Hapmap is generated by the DiscoverySNPCallerPlugin. For species with no reference sequence available, a network-based algorithm (UNEAK) is used for genotype calling.

\subsubsection{Processing of hapmap data}

Raw SNP data output by the TASSEL-GBS pipeline are further filtered for mapping purposes. SNPs with minor allele frequency less than 0.05 and proportion of missing genotypes greater than $40 \%$ are removed from further analysis. In early-generation (F2, Backcross, DH, RIL) mapping populations, SNPs that are closely located on a chromosome often do not detect different recombination events and thus provide essentially redundant information. In order to reduce the number of redundant SNPs in studies where strong linkage disequilibrium necessitates only 5-10 cM resolution, we merge SNPs of which pairwise 
$\mathrm{LD}\left(\mathrm{r}^{2}\right)$ is greater than 0.9 . The consensus genotypes are derived in a manner minimizing missing genotypes. Closely located SNPs are further merged if the Pearson's correlation between them is larger than 0.95 . For bi-parental populations, the missing genotype of one parental line can be imputed by offspring genotypes if the genotype of the other parent at the locus is known. After these filtering steps, SNP data are used for genetic mapping.

Heterozygous calling is a great challenge for GBS studies with low read coverage. Theoretically, at a single locus $6 \times$ read coverage is needed to call a heterozygous genotype with confidence larger than 0.95 if a binomial distribution is assumed in a diploid species. However, most GBS protocols including ours focus on low-coverage sequence data, with few individual loci having $6 \times$ read coverage. It is a trade-off between the number of filtered SNPs and the confidence of heterozygous genotype calling. In our study, we use genotypes that have at least $2 \times$ read coverage, which results in many missing values. However, missing values can be alleviated by some imputation steps comparing SNP data to haplotypes or marker orders. Different tools such as Beagle [27], Impute2 [28], MaCH [29], and minimac2 [30] have been implemented in various genetic studies and improved the quality of SNP datasets. 'Bin mapping' [7] is a good example to infer missing genotypes using marker orders. For outcrossing species, the Mascov algorithm which corrects genotype errors or infers missing genotypes based on linkage phase and configuration was recently proposed and dramatically enhanced the power of detecting recombination events [31]. It is not practical to re-sequence GBS libraries to obtain missing values largely due to cost. A variety of imputation methods may be able to save much time and effort to reach the desired SNP density.

\subsubsection{Equations that calculate expected number of reads}

Determining read coverage is an important step in the experimental design of GBS. Here, we formulate an empirical equation that can be used to estimate the minimum number of reads to obtain a targeted number of SNPs. 
Noting that most GBS approaches use restriction digests, the expected number of restriction fragments in a genome is routinely calculated by:

$$
\frac{N}{4^{n}}
$$

Where $\mathrm{N}$ is a genome size (bp) and $\mathrm{n}$ is the length of enzyme recognition site (bp).

Next, an expected number of SNPs within these restriction fragments can be estimated by:

$$
E_{(\# S N P)}=\frac{N r p}{4^{n}}
$$

Where $\mathrm{r}$ is the length of read (bp) and $\mathrm{p}$ is the per-nucleotide polymorphism rate.

The coverage $(C)$, or number of sequenced restriction fragments per polymorphic site can be calculated by:

$$
C=M u b
$$

Where $\mathrm{M}$ is the total number of individuals, $\mathrm{u}$ is a call rate (the number of genotyped individuals divided by the total number of individuals), and $\mathrm{b}$ is a ploidy number.

Consequently, the minimum number of reads estimated to be required for a particular experiment $(T)$ can be estimated by:

$$
T=\frac{N C}{4^{n}}
$$

If we combine the above equations to integrate the expected number of SNPs $\left[E_{(\# S N P)}\right]$ : 


$$
T=\frac{E_{(\# S N P)} M u b}{r p}
$$

Given some prior GBS data from a genome, one can routinely adjust for sampling bias, altering the number of restriction fragments $\left(\mathrm{N} / 4^{\mathrm{n}}\right)$ due to non-random occurrence of DNA sequence motifs, methylation sensitivity of restriction enzymes, or technical reasons such as incomplete digestion or gel size selection. For example, in test data from an F1 mapping population (186 individuals total) derived from an interspecific cross between two diploids, Miscanthus sinensis and M. sacchariflorus, where the observed number of SNPs was 52,683, $\mathrm{M}=186, \mathrm{~b}=2, \mathrm{r}=64, \mathrm{p}=0.005$, and $\mathrm{u}=0.35$. With no sampling bias, the observed number of SNPs $(52,683)$ would be expected from $T$ (the minimum number of reads) of 21,435,395. However, the observed number of SNPs actually required 55,396,954 reads to obtain. Thus, a coefficient $(\rho=55,396,954 / 21,435,395=2.58)$ that accounts for sampling bias should be added to the function.

$$
T=\frac{2.58 E_{(\# S N P)} M u b}{r p}
$$

As a result, we can calculate the minimum number of reads to obtain a certain number of SNPs. For example, if we want to obtain 10,000 SNPs in a Miscanthus population with $\mathrm{M}=100, \mathrm{~b}=2, \mathrm{r}=64, \mathrm{p}=0.005$, and $\mathrm{u}=0.35$, the minimum number of reads to obtain 10,000 SNPs is as follows:

$$
T=\frac{2.58 \times 10,000 \times 100 \times 0.35 \times 2}{64 \times 0.005}=5,643,750(\text { reads })
$$

A reliable coefficient $(\rho)$ to account for sampling bias must be empirically determined for each species, because every plant has a unique genome structure and methylation profile.

\subsection{Sorghum}


Application of GBS to sorghum has been routine in our group because it has a well-established reference genome with a relatively small genome size (ca. $700 \mathrm{Mb}$ ) and a minimum of gene and low-copy sequence duplication [32]. Our pilot GBS study of sorghum was conducted with an $\mathrm{F}_{6}$ population (RIL) from an intraspecific cross between two S. bicolor cultivars, BTx623 and IS6320C. RILs are particularly facile for GBS because of their high homozygosity, minimizing heterogygote genotyping errors caused by low read depth. The pilot study was performed by a single MiSeq run (ca. 25 million reads in total) with 90 mapping individuals plus parents (three redundant samples each), resulting in a total of 576 SNPs genetically mapped with the aid of the reference genome (Fig. 3A). Genotype calling from GBS agrees very well with SSR marker genotypes (data not shown). The orders of SNPs are also well-aligned to the physical map of sorghum, indicating that GBS is well suited for this population with much higher resolution and less intensive lab work (Fig. 3B). GBS provides a rapid and robust tool for genotyping largely-homozygous sorghum populations.

\subsection{Brassica}

Brassica oleracea, including numerous vegetable crops [33], is of great economic importance and remarkable morphological diversity, including Brussels sprouts (var. gemmifera), broccoli (var. italica), cauliflower (var. botrytis), Chinese kale (var. alboglabra), kale and collards (var. acephala), kohlrabi (var. gongylodes), cabbage (var. capitata) and rapid cycling lines [34, 35]. Toward dissecting phenotypic variation among morphotypes, we genotyped two $\mathrm{BC}_{4} \mathrm{~F}_{1}$ populations by $\mathrm{GBS}$ to identify introgression from donor parent in each line. The two populations respectively involved inbred strains of cabbage (Badger Inbred) and cauliflower (Orange) as donor parents and an inbred rapid cycling line, TO1434 as recurrent parent. In the cauliflower $\mathrm{BC}_{4} \mathrm{~F}_{1}$ population, 89 lines were genotyped by $693 \mathrm{SNP}$ markers, identifying a total of 164 introgressed segments, with zero to seven introgressed segments (averaging 2.4) per line (Table 3). In cabbage $\mathrm{BC}_{4} \mathrm{~F}_{1}$ population, 75 lines were genotyped by $641 \mathrm{SNP}$ markers, finding 162 introgressed segments, with zero to seven (averaging 2.6) per line (Table 3). The respective average 
of the two populations, 2.4 and 2.6 segments, closely approximate the a priori expectation of 2.5 introgressed segments per line in the $\mathrm{BC}_{4} \mathrm{~F}_{1}$ generation [36].

\subsection{Cotton}

We are aiming to develop two reciprocal sets of NILs by introgression between two tetraploid species, Acala Maxxa (Gossypium hirsutum) and Pima S6 (Gossypium barbadense) via repeated back crossing and single seed descent. Using GBS screening to determine genomic composition of $\mathrm{BC}_{4} \mathrm{~F}_{1}$ plants and design strategies to obtain NILs has been very time-efficient. In the BC4F1 population with Acala Maxxa as recurrent parent, we used 956 SNPs to genotype 39 individuals which resulted in finding a total of 106 introgressed regions and on average, one BC4F1 plant retained 2.7 introgressed regions. For the BC4F1 population with Pima S6 as recurrent parent, we genotyped 39 individuals with 914 SNPs and found a total of 114 introgression segments (averaging 2.9 introgressions). The average number of introgressions $(\sim 3)$ in both populations was somewhat less than randomly expected numbers of introgressions (4) in similar genotypes, consistent with widespread evidence of less-than expected rates of introgression in prior studies [37]. We have tested 30 of these introgression regions randomly by using one to two SSRs from each region in $\mathrm{BC}_{4} \mathrm{~F}_{1}$ progenies and results showed $90 \%$ consistency (27 of 30 regions). We are planning to integrate at least 2 SSRs in each introgression region, which will facilitate selection of NILs and simultaneously increase the marker density for high resolution mapping. The development of genome-wide NILs will solve three purposes simultaneously; identification of QTL, fine mapping, and incorporation of the QTL in the elite germplasm [38].

\subsection{Miscanthus}

Miscanthus is a promising feedstock for lignocellulosic bioethanol production. Current cultivars (M.× giganteus) are largely triploid hybrids between tetraploid (M. saccahriflorus) and diploid (M. sinensis) progenitors. The species are characterized by their large genome sizes (ca. $4.3 \sim 6.8 \mathrm{~Gb}$ ) [39] and outcrossing nature. We previously published genetic maps of two diploid species, M. sacchariflorus 
Robustus and M. sinensis with about 600 SSRs [40]. Due to their high heterozygosity, the two-way pseudotestcross model [23] was used with an $\mathrm{F}_{1}$ mapping population. The same population is very challenging for GBS application in many ways. For example, the genome of Miscanthus was doubled about 3.5 million years ago (MYA) by allopolyploidy and diploidized after its divergence from the Saccharum lineage (ca. 3.1 MYA) [41], although still containing many paralogous sequences that can interfere with SNP genotyping in the absence of a reference genome. Most importantly, it has a large genome size which requires multiple MiSeq runs to achieve minimum read depth to discover SNPs. The low read depth in this population is a particularly serious problem in that the accurate distinction of heterozygotes from homozygotes is a key in mapping outcrossing species. With low sequence coverage, heterozygotes are usually misread as homozygotes. To resolve this problem, we adopted a new imputation method specifically developed for outcrossing species. The Mascov algorithm [31] efficiently predicts missing genotypes based on SNP orders and their genotype/phase configurations. Currently, we are trying to integrate ca. 1,000 SNPs with existing SSR maps to find QTLs with biomass-related traits. The enhanced genetic/QTL maps will be important assets for MAS in Miscanthus breeding efforts.

\section{Future perspectives and concluding remarks}

GBS utilizes NGS data to identify allelic combinations based on SNPs. The sequencing read depth that proves necessary for a particular experimental design can be affected by some biological factors of a target species, including: (1) Genome size, (2) Genome complexity, (3) Ploidy, and (4) Expected heterozygosity. Poor coverage of specific loci may cause excessive missing values, compromising the accuracy of genetic studies. Imputation of missing values can in part relieve the adverse effect of missing genotypes; however, the fundamental solution may be in the adjustment of technical factors. For example, read depth per locus can be reinforced by reduced numbers of genotypes per library, use of rare cutting restriction enzymes, double digestion, and multiple sequencing runs for a library. Since the biggest strength of GBS is cost-effectiveness, it will be crucial to find some middle ground among the optimal 
number of multiplexed samples, the choice of restriction enzymes, and sequence capacity based on the genome size of a target plant.

Despite the remaining challenges, GBS is a fascinating approach to fulfill researchers' needs. Sequencing costs continue to drop, and computational data processing is becoming more routine. GBS does not require a priori genomic knowledge of the species of interest, making it an ideal tool for orphan crops with scarce genomic resources. Some economically important crops with complex or large genomes (e.g. sugarcane) may be major beneficiaries of this advanced technology. MAS through abundant SNPs generated by GBS will facilitate selecting target plants as well as scaling up breeding programs. As exemplified in pilot studies, GBS permits one to generate a robust tool set for plant breeding with speed and efficiency. In addition to breeding applications, GBS has the potential to answer a number of genomic questions in junction with its plentiful sequence information.

\section{Acknowledgements}

We appreciate comments and suggestions from numerous current and past members of the Plant Genome Mapping Laboratory, and financial support from the US National Science Foundation $(0817707,1127755)$ and the DOE-USDA Plant Feedstock Program (112786, 129042 to AHP).

\section{References}

[1] P. Gerland, A.E. Raftery, H. Ševčíková, N. Li, D. Gu, T. Spoorenberg, L. Alkema, B.K. Fosdick, J. Chunn, N. Lalic, G. Bay, T. Buettner, G.K. Heilig, J. Wilmoth, World population stabilization unlikely this century, Science, 346 (2014) 234-237.

[2] Arabidopsis Genome Initiative, Analysis of the genome sequence of the flowering plant Arabidopsis thaliana, Nature, 408 (2000) 796-815.

[3] J. Yu, S. Hu, J. Wang, G.K. Wong, S. Li, B. Liu, Y. Deng, L. Dai, Y. Zhou, X. Zhang, M. Cao, J. Liu, J. Sun, J. Tang, Y. Chen, X. Huang, W. Lin, C. Ye, W. Tong, L. Cong, J. Geng, Y. Han, L. Li, W. Li, 
G. Hu, X. Huang, W. Li, J. Li, Z. Liu, L. Li, J. Liu, Q. Qi, J. Liu, L. Li, T. Li, X. Wang, H. Lu, T. Wu, M. Zhu, P. Ni, H. Han, W. Dong, X. Ren, X. Feng, P. Cui, X. Li, H. Wang, X. Xu, W. Zhai, Z. Xu, J. Zhang, S. He, J. Zhang, J. Xu, K. Zhang, X. Zheng, J. Dong, W. Zeng, L. Tao, J. Ye, J. Tan, X. Ren, X. Chen, J. He, D. Liu, W. Tian, C. Tian, H. Xia, Q. Bao, G. Li, H. Gao, T. Cao, J. Wang, W. Zhao, P. Li, W. Chen, X. Wang, Y. Zhang, J. Hu, J. Wang, S. Liu, J. Yang, G. Zhang, Y. Xiong, Z. Li, L. Mao, C. Zhou, Z. Zhu, R. Chen, B. Hao, W. Zheng, S. Chen, W. Guo, G. Li, S. Liu, M. Tao, J. Wang, L. Zhu, L. Yuan, H. Yang, A draft sequence of the rice genome (Oryza sativa L. ssp. indica), Science, 296 (2002) 79-92.

[4] R.J. Elshire, J.C. Glaubitz, Q. Sun, J.A. Poland, K. Kawamoto, E.S. Buckler, S.E. Mitchell, A robust, simple genotyping-by-sequencing (GBS) approach for high diversity species, PLoS One, 6 (2011) e19379.

[5] N.A. Baird, P.D. Etter, T.S. Atwood, M.C. Currey, A.L. Shiver, Z.A. Lewis, E.U. Selker, W.A. Cresko, E.A. Johnson, Rapid SNP discovery and genetic mapping using sequenced RAD markers, PLoS One, 3 (2008) e3376.

[6] P. Andolfatto, D. Davison, D. Erezyilmaz, T.T. Hu, J. Mast, T. Sunayama-Morita, D.L. Stern, Multiplexed shotgun genotyping for rapid and efficient genetic mapping, Genome Research, 21 (2011) 610-617.

[7] J.A. Poland, P.J. Brown, M.E. Sorrells, J.L. Jannink, Development of high-density genetic maps for barley and wheat using a novel two-enzyme genotyping-by-sequencing approach, PLoS One, 7 (2012) e32253.

[8] H.T. Truong, A.M. Ramos, F. Yalcin, M. de Ruiter, H.J. van der Poel, K.H. Huvenaars, R.C. Hogers, L.J. van Enckevort, A. Janssen, N.J. van Orsouw, M.J. van Eijk, Sequence-based genotyping for marker discovery and co-dominant scoring in germplasm and populations, PLoS One, 7 (2012) e37565. 
[9] E. Stolle, R.F. Moritz, RESTseq - efficient benchtop population genomics with RESTriction Fragment SEQuencing, PLoS One, 8 (2013) e63960.

[10] J. Monson-Miller, D.C. Sanchez-Mendez, J. Fass, I.M. Henry, T.H. Tai, L. Comai, Reference genome-independent assessment of mutation density using restriction enzyme-phased sequencing, BMC Genomics, 13 (2012) 72.

[11] X. Huang, Q. Feng, Q. Qian, Q. Zhao, L. Wang, A. Wang, J. Guan, D. Fan, Q. Weng, T. Huang, G. Dong, T. Sang, B. Han, High-throughput genotyping by whole-genome resequencing, Genome Research, 19 (2009) 1068-1076.

[12] X. Huang, Y. Zhao, X. Wei, C. Li, A. Wang, Q. Zhao, W. Li, Y. Guo, L. Deng, C. Zhu, D. Fan, Y. Lu, Q. Weng, K. Liu, T. Zhou, Y. Jing, L. Si, G. Dong, T. Huang, T. Lu, Q. Feng, Q. Qian, J. Li, B. Han, Genome-wide association study of flowering time and grain yield traits in a worldwide collection of rice germplasm, Nature Genetics, 44 (2012) 32-39.

[13] J. Xu, Q. Zhao, P. Du, C. Xu, B. Wang, Q. Feng, Q. Liu, S. Tang, M. Gu, B. Han, G. Liang, Developing high throughput genotyped chromosome segment substitution lines based on population whole-genome re-sequencing in rice (Oryza sativa L.), BMC Genomics, 11 (2010) 656.

[14] C.P. Van Tassell, T.P. Smith, L.K. Matukumalli, J.F. Taylor, R.D. Schnabel, C.T. Lawley, C.D. Haudenschild, S.S. Moore, W.C. Warren, T.S. Sonstegard, SNP discovery and allele frequency estimation by deep sequencing of reduced representation libraries, Nature Methods, 5 (2008) 247-252.

[15] J.A. Poland, T.W. Rife, Genotyping-by-Sequencing for Plant Breeding and Genetics, The Plant Genome, 5 (2012) 92-102.

[16] S. Deschamps, V. Llaca, G.D. May, Genotyping-by-Sequencing in Plants, Biology, 1 (2012) 460483.

[17] J. He, X. Zhao, A. Laroche, Z.X. Lu, H. Liu, Z. Li, Genotyping-by-sequencing (GBS), an ultimate marker-assisted selection (MAS) tool to accelerate plant breeding, Frontiers in Plant Science, 5 (2014) 484. 
[18] B. Langmead, C. Trapnell, M. Pop, S.L. Salzberg, Ultrafast and memory-efficient alignment of short DNA sequences to the human genome, Genome Biology, 10 (2009) R25.

[19] H. Li, R. Durbin, Fast and accurate short read alignment with Burrows-Wheeler transform, Bioinformatics, 25 (2009) 1754-1760.

[20] J. Catchen, P.A. Hohenlohe, S. Bassham, A. Amores, W.A. Cresko, Stacks: an analysis tool set for population genomics, Molecular Ecology, 22 (2013) 3124-3140.

[21] J.C. Glaubitz, T.M. Casstevens, F. Lu, J. Harriman, R.J. Elshire, Q. Sun, E.S. Buckler, TASSELGBS: a high capacity genotyping by sequencing analysis pipeline, PLoS One, 9 (2014) e90346.

[22] F. Lu, A.E. Lipka, J. Glaubitz, R. Elshire, J.H. Cherney, M.D. Casler, E.S. Buckler, D.E. Costich, Switchgrass genomic diversity, ploidy, and evolution: novel insights from a network-based SNP discovery protocol, PLoS Genetics, 9 (2013) e1003215.

[23] D. Grattapaglia, R. Sederoff, Genetic linkage maps of Eucalyptus grandis and Eucalyptus urophylla using a pseudo-testcross: Mapping strategy and RAPD markers, Genetics, 137 (1994) 1121-1137.

[24] E.A. Stone, J.F. Ayroles, Modulated modularity clustering as an exploratory tool for functional genomic inference, PLoS Genetics, 5 (2009) e1000479.

[25] J.L. Bennetzen, J. Schmutz, H. Wang, R. Percifield, J. Hawkins, A.C. Pontaroli, M. Estep, L. Feng, J.N. Vaughn, J. Grimwood, J. Jenkins, K. Barry, E. Lindquist, U. Hellsten, S. Deshpande, X. Wang, X. Wu, T. Mitros, J. Triplett, X. Yang, C.Y. Ye, M. Mauro-Herrera, L. Wang, P. Li, M. Sharma, R. Sharma, P.C. Ronald, O. Panaud, E.A. Kellogg, T.P. Brutnell, A.N. Doust, G.A. Tuskan, D. Rokhsar, K.M. Devos, Reference genome sequence of the model plant Setaria, Nature Biotechnology, 30 (2012) $555-561$.

[26] Y.F. Huang, J.A. Poland, C.P. Wight, E.W. Jackson, N.A. Tinker, Using genotyping-by-sequencing (GBS) for genomic discovery in cultivated oat, PLoS One, 9 (2014) e102448. 
[27] S.R. Browning, B.L. Browning, Rapid and accurate haplotype phasing and missing-data inference for whole-genome association studies by use of localized haplotype clustering, AmericanJournal of Human Genetics, 81 (2007) 1084-1097.

[28] J. Marchini, B. Howie, S. Myers, G. McVean, P. Donnelly, A new multipoint method for genomewide association studies by imputation of genotypes, Nature Genetics, 39 (2007) 906-913.

[29] Y. Li, C.J. Willer, J. Ding, P. Scheet, G.R. Abecasis, MaCH: using sequence and genotype data to estimate haplotypes and unobserved genotypes, Genetic Epidemiology, 34 (2010) 816-834.

[30] B. Howie, C. Fuchsberger, M. Stephens, J. Marchini, G.R. Abecasis, Fast and accurate genotype imputation in genome-wide association studies through pre-phasing, Nature Genetics, 44 (2012) 955959.

[31] J.A. Ward, J. Bhangoo, F. Fernandez-Fernandez, P. Moore, J.D. Swanson, R. Viola, R. Velasco, N. Bassil, C.A. Weber, D.J. Sargent, Saturated linkage map construction in Rubus idaeus using genotyping by sequencing and genome-independent imputation, BMC Genomics, 14 (2013) 2.

[32] A.H. Paterson, J.E. Bowers, R. Bruggmann, I. Dubchak, J. Grimwood, H. Gundlach, G. Haberer, U. Hellsten, T. Mitros, A. Poliakov, J. Schmutz, M. Spannagl, H. Tang, X. Wang, T. Wicker, A.K. Bharti, J. Chapman, F.A. Feltus, U. Gowik, I.V. Grigoriev, E. Lyons, C.A. Maher, M. Martis, A. Narechania, R.P. Otillar, B.W. Penning, A.A. Salamov, Y. Wang, L. Zhang, N.C. Carpita, M. Freeling, A.R. Gingle, C.T. Hash, B. Keller, P. Klein, S. Kresovich, M.C. McCann, R. Ming, D.G. Peterson, M. Rahman, D. Ware, P. Westhoff, K.F. Mayers, J. Messing, D.S. Rokhsar, The Sorghum bicolor genome and the diversification of grasses, Nature, 457 (2009) 551-556.

[33] G. Rakow, Species origin and economic importance of Brassica, in: Brassica, Springer, 2004, pp. 3 11.

[34] G.R. Dixon, Vegetable brassicas and related crucifers / Geoffrey R Dixon, Wallingford, UK ; Cambridge, MA : CABI Pub., c2007., 2007.

[35] P.H. Williams, C.B. Hill, Rapid-cycling populations of Brassica, Science, 232 (1986) 1385-1389. 
[36] W.D. Hanson, Early Generation Analysis of Lengths of Heterozygous Chromosome Segments around a Locus Held Heterozygous with Backcrossing or Selfing, Genetics, 44 (1959) 833-837.

[37] C.X. Jiang, P.W. Chee, X. Draye, P.L. Morrell, C.W. Smith, A.H. Paterson, Multilocus interactions restrict gene introgression in interspecific populations of polyploid Gossypium (cotton), Evolution; International Journal of Organic Evolution, 54 (2000) 798-814.

[38] Y. Eshed, D. Zamir, A genomic library of Lycopersicon pennellii in L. esculentum: A tool for fine mapping of genes, Euphytica, 79 (1994) 175-179.

[39] A.L. Rayburn, J. Crawford, C.M. Rayburn, J.A. Juvik, Genome size of Three Miscanthus species, Plant Molecular Biology Reporter, 27 (2009) 184-188.

[40] C. Kim, D. Zhang, S.A. Auckland, L.K. Rainville, K. Jakob, B. Kronmiller, E.J. Sacks, M. Deuter, A.H. Paterson, SSR-based genetic maps of Miscanthus sinensis and M. sacchariflorus, and their comparison to sorghum, Theoretical and Applied Genetics, 124 (2012) 1325-1338.

[41] C. Kim, X. Wang, T.H. Lee, K. Jakob, G.J. Lee, A.H. Paterson, Comparative Analysis of Miscanthus and Saccharum Reveals a Shared Whole-Genome Duplication but Different Evolutionary Fates, The Plant Cell, 26 (2014) 2420-2429.

[42] B.K. Peterson, J.N. Weber, E.H. Kay, H.S. Fisher, H.E. Hoekstra, Double digest RADseq: an inexpensive method for de novo SNP discovery and genotyping in model and non-model species, PLoS One, 7 (2012) e37135.

[43] M. Mascher, S. Wu, P.S. Amand, N. Stein, J. Poland, Application of genotyping-by-sequencing on semiconductor sequencing platforms: a comparison of genetic and reference-based marker ordering in barley, PLoS One, 8 (2013) e76925. 


\section{Figure legends}

Fig. 1. Two types of common genotyping mistake caused by sequencing errors (A) and low read depth (B). Genotyping errors are commonly associated with the mis-identification of heterozygotes from homozygotes.

Fig. 2. Complication of GBS in tetraploid species. In autotetraploids without a reference genome (A), allelic dosage cannot be evaluated under low coverage data. In allotetraploids without a reference genome (B), allelic variations between subgenomes cannot be identified when the similarity of homeologs is fairly high.

Fig. 3. A sorghum genetic map with 90 RIL individuals (A). A total of 576 SNPs were mapped by a single MiSeq run with a GBS library. The genetic map is well-aligned to the sorghum physical map (B). Each physical map does not cover the entire chromosome because only partial fragments which are consistent with marker locations are represented. 
Table 1. Summary of commercially-available SNP genotyping platforms

\begin{tabular}{|c|c|c|c|c|c|}
\hline Platform & Provider & Assay type & Technology & Throughput & Multiplexing \\
\hline Taqman & Applied Biosystems & PCR & Taqman probe & 1536/day & $\sim 256$ \\
\hline SNPlex & Applied Biosystems & PCR & Capillary electrophoresis & 1536/3 days & $\sim 48$ \\
\hline BioMark HD & Fluidigm & PCR & Microfluid-based chips & $\sim 96 / 3$ hours & $\sim 96$ \\
\hline KASPar & LGC & PCR & FRET quenching oligos & 96/day & Not available \\
\hline Axiom Biobank & Affymetrix & Hybridization & Oligo nucleotide array & $\sim 96 / 5$ days & $\sim 650 \mathrm{~K}$ \\
\hline Infinium II & Illumina & Hybridization & Bead array & 128/5 days & $\sim 700 \mathrm{~K}$ \\
\hline GoldenGate & Ilumina & Primer extension & Bead array & $\sim 172 / 3$ days & $\sim 1536$ \\
\hline ¡Plex & Sequenome & Primer extension & Mass spectrometry & 3840/2.5 days & $\sim 40$ \\
\hline
\end{tabular}


Table 2. Representative GBS protocols published in peer-reviewed journals

\begin{tabular}{|c|c|c|c|c|c|c|}
\hline Method & $\begin{array}{l}\text { Restriction } \\
\text { enzyme }\end{array}$ & Insert size & Barcodes & $\begin{array}{l}\text { Sequencing } \\
\text { platform }\end{array}$ & $\begin{array}{l}\text { Sequencing } \\
\text { mode }\end{array}$ & Reference \\
\hline $\begin{array}{l}\text { RAD-seq (Restriction association DNA } \\
\text { sequencing) }\end{array}$ & Sbfl or EcoRI & Size-selection & $\sim 96$ & Illumina & Paired-end & [5] \\
\hline MSG (Multiplex shotgun genotyping ) & Msel & Size-selection & $\sim 384$ & Illumina & Single-end & [6] \\
\hline GBS (Genotype by sequencing) & ApeKI & $<350 \mathrm{bp}$ & $\sim 384$ & Illumina & Paired-end & [4] \\
\hline Double-digested RAD-seq & EcoRI and Mspl & Size-selection & $\sim 48$ & Illumina & Paired-end & [42] \\
\hline Double-digested GBS & Pstl and Mspl & $<350$ bp & $\sim 384$ & Illumina & Paired-end & [7] \\
\hline Ion Torrent GBS & Pstl and Mspl & $<350 \mathrm{bp}$ & $\sim 384$ & Ion Torrent & Paired-end & [43] \\
\hline SBG ( Sequence-based genotyping) & $\begin{array}{l}\text { EcoRI and Msel } \\
\text { Pstl and Msel }\end{array}$ & Size-selection & $\sim 32$ & Illumina & Paired-end & [8] \\
\hline $\begin{array}{l}\text { REST-seq (Restriction fragment } \\
\text { sequencing) }\end{array}$ & Taql and Trul & Size-selection & $\sim 305$ & Ion Torrent & Paired-end & [9] \\
\hline $\begin{array}{l}\text { Restriction enzyme sequence } \\
\text { comparative analysis }\end{array}$ & Msel or NlallI & Size-selection & $\sim 96$ & Illumina & Paired-end & {$[10]$} \\
\hline
\end{tabular}


Table 3. Summary of cauliflower $\mathrm{BC}_{4} \mathrm{~F}_{2}$ and cabbage $\mathrm{BC}_{4} \mathrm{~F}_{1}$ genotypes

\begin{tabular}{rcr}
\hline Number of introgressed & Cauliflower $\mathrm{BC}_{4} \mathrm{~F}_{2}$ & Cabbage $\mathrm{BC}_{4} \mathrm{~F}_{1}$ \\
\cline { 2 - 3 } segments & Number of lines & Number of lines \\
\hline Missing & 7 & 12 \\
0 & 7 & 4 \\
1 & 26 & 10 \\
2 & 24 & 19 \\
3 & 17 & 13 \\
4 & 4 & 12 \\
5 & 2 & 4 \\
6 & 1 & 0 \\
7 & 1 & 1 \\
\hline
\end{tabular}


Fig. 1.

A. Sequencing error

\section{Correct genotyping - homozygous for parent 1}

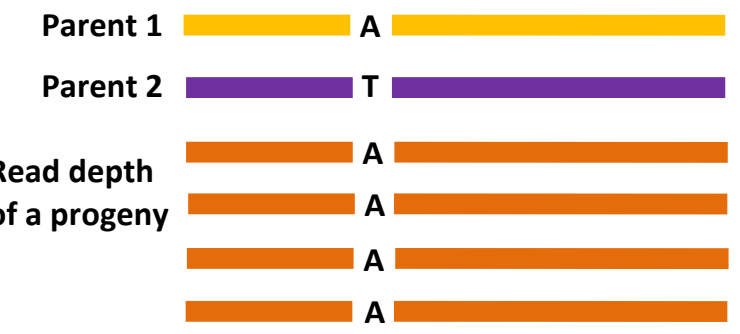

B. Low coverage

Correct genotyping - heterozygous at $4 \mathrm{X}$ coverage

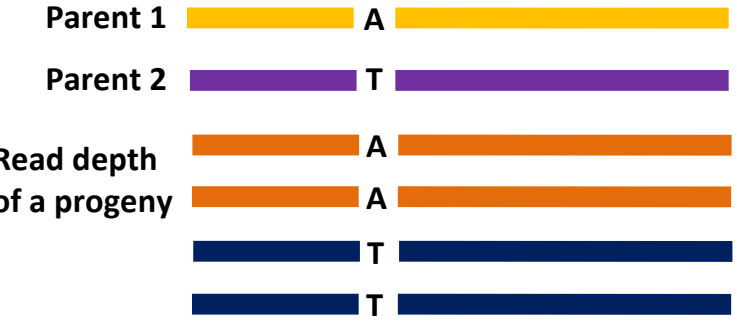

Incorrect - genotyped as heterozygous due to sequencing error

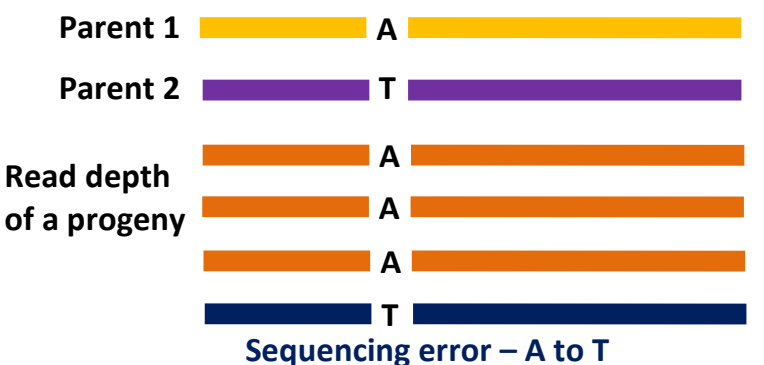

Sequencing error $-\mathrm{A}$ to $\mathrm{T}$

Incorrect - homozygous at $2 X$ coverage

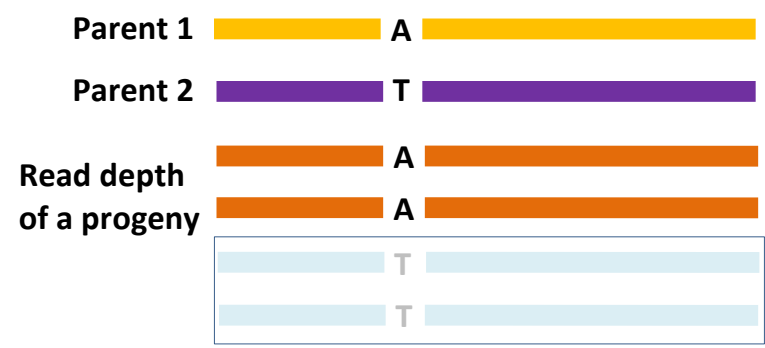

' $T$ ' alleles are not sequenced by chance at $2 X$ coverage 
Fig. 2.

\section{A. Autotetraploids - SNP alignment}

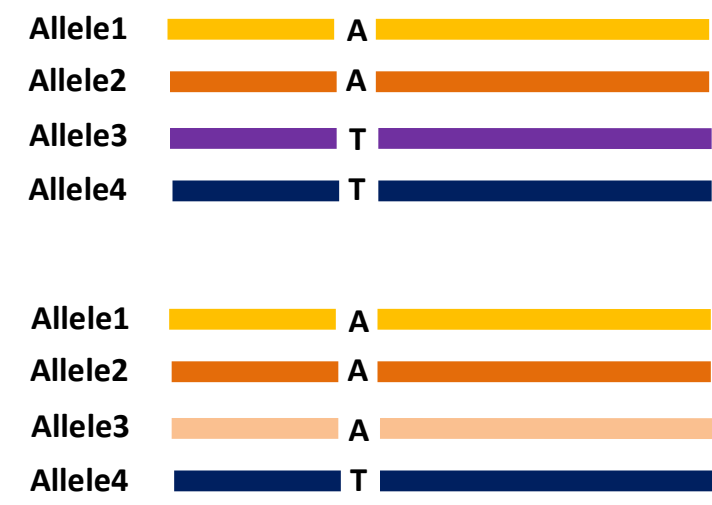

\section{B. Allotetraploid - SNP alignment}

Locus
Ref sub A

Both cases are genotyped as heterozygous

despite the difference in allelic dosage 
Fig. 3.

A

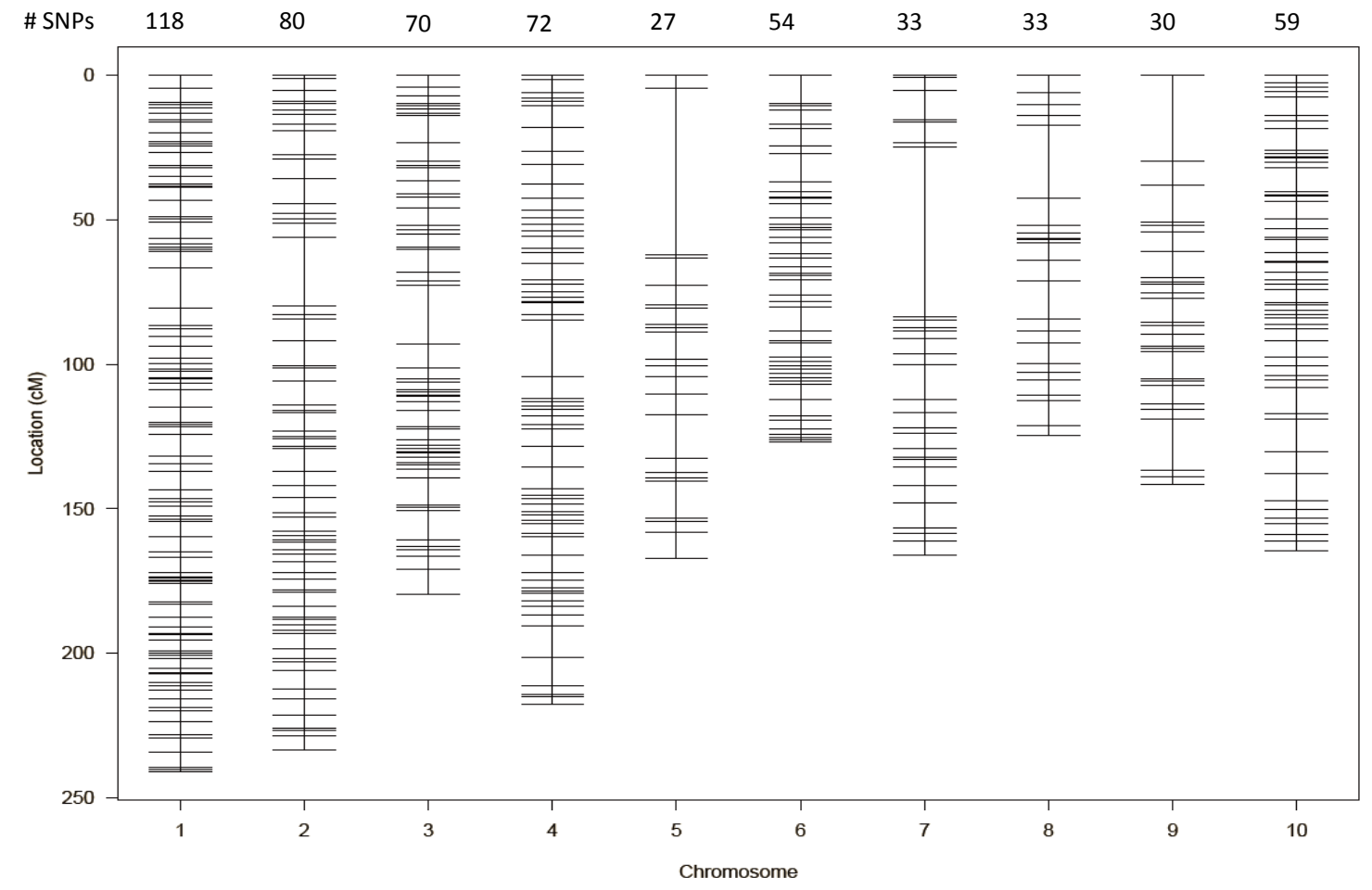

B

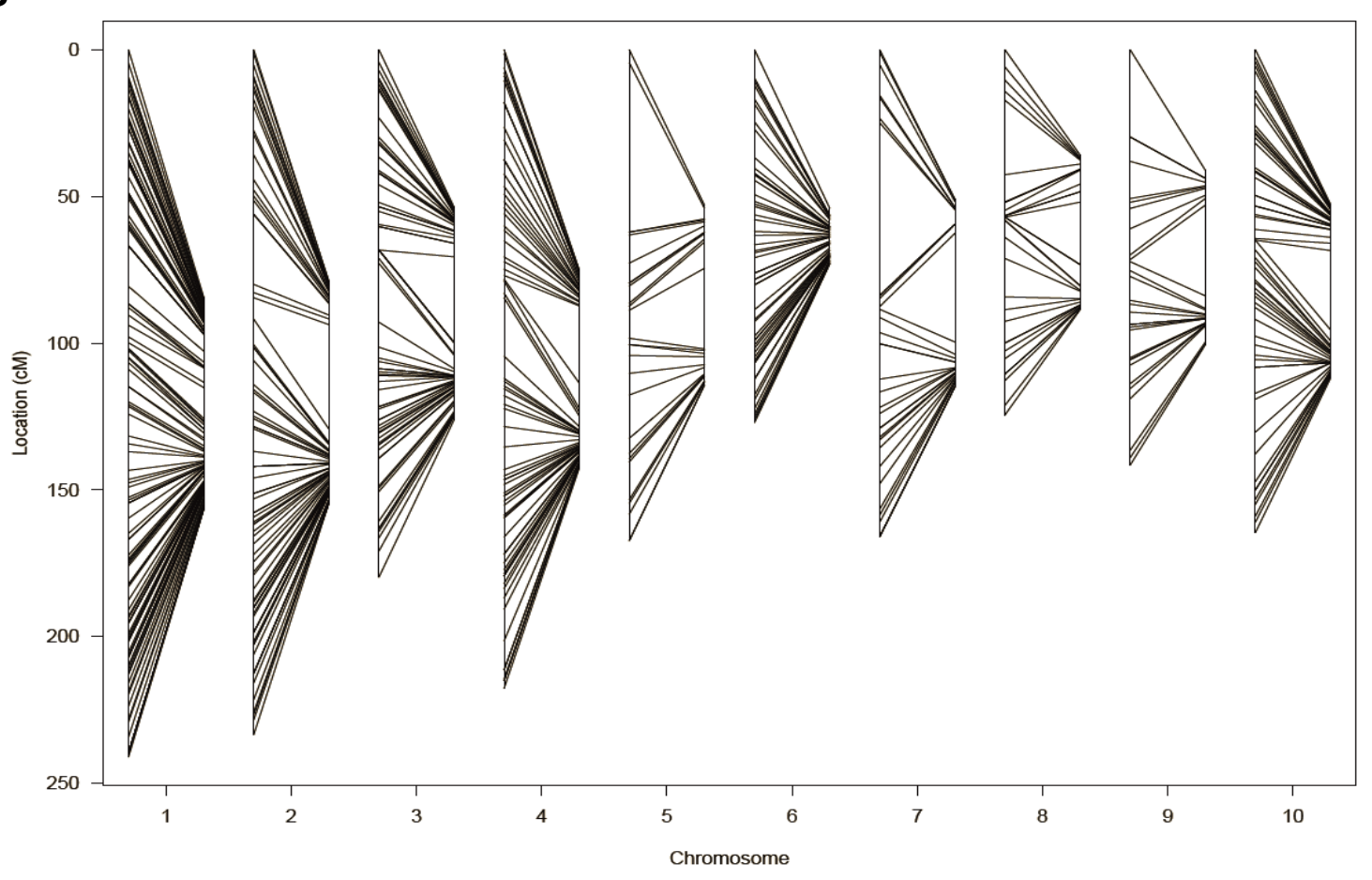

